\title{
Image quality and artifacts in automated breast ultrasonography
}

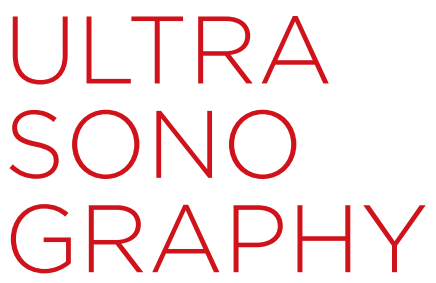

\author{
Sung Hun Kim \\ Department of Radiology, Seoul St. Mary's Hospital, College of Medicine, The Catholic \\ University of Korea, Seoul, Korea
}

Three-dimensional automated breast ultrasonography (ABUS) has been approved for screening studies as an adjunct to mammography. ABUS provides proper orientation and documentation, resulting in better reproducibility. Optimal image quality is essential for a proper diagnosis, and high-quality images should be ensured when ABUS is used in clinical settings. Image quality in ABUS is highly dependent on the acquisition procedure. Artifacts can interfere with the visibility of abnormalities, reduce the overall image quality, and introduce clinical and technical problems. Nipple shadow and reverberation artifacts are some of the artifacts frequently encountered in ABUS. Radiologists should be familiar with proper image acquisition techniques and possible artifacts in order to acquire high-quality images.

Keywords: Ultrasonography, mammary; Artifacts; Radiologists; Mass screening;

Early detection of cancer

Received: April 2, 2018

Revised: July 10, 2018

Accepted: July 14, 2018

Correspondence to:

Sung Hun Kim, MD, Department of

Radiology, Seoul St. Mary's Hospital,

College of Medicine, The Catholic

University of Korea, 222 Banpo-daero,

Seocho-gu, Seoul 06591, Korea

Tel. +82-2-2258-6250

Fax. +82-2-2258-1457

E-mail: rad-ksh@catholic.ac.kr

\section{Introduction}

Mammography is the only imaging modality that has been proven to reduce mortality from breast cancer [1]. However, the sensitivity of mammography in dense breasts is poor [2]. In the United States, state-level legislation requires radiologists to inform patients of the aforementioned disadvantage of mammography and the possible need for supplemental screening modalities, such as ultrasonography, in patients with dense breasts [3].

Three-dimensional automated breast ultrasonography (ABUS) has been approved in the United States and Europe to serve as a screening tool for breast cancer. ABUS is used as an adjunct to mammography and for screening asymptomatic women with dense breasts [4].

ABUS is designed to overcome the shortfalls associated with hand-held US (HHUS), such as operator dependency and lack of reproducibility. It provides proper orientation and documentation, resulting in better reproducibility, and is also reliable for follow-up studies. For technologists, the operation of ABUS is user-friendly, with no need for prolonged training. In addition to this, it is timeefficient for radiologists [5-8].

Optimal image quality is essential for a proper diagnosis, and an imaging system should ensure high-quality images before approval for use in clinical practice [9]. Image quality can be impacted by the image acquisition process [9]. Artifacts can interfere with the visibility of abnormalities, reduce overall image quality, and adversely affect the reliability of an imaging system [10]. Radiologists

This is an Open Access article distributed under the terms of the Creative Commons Attribution NonCommercial License (http://creativecommons.org/ licenses/by-nc/3.0/) which permits unrestricted noncommercial use, distribution, and reproduction in any medium, provided the original work is properly cited.

Copyright (C) 2019 Korean Society of Ultrasound in Medicine (KSUM)

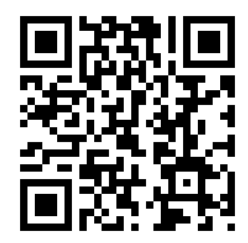

How to cite this article:

Kim SH. Image quality and artifacts in automated breast ultrasonography. Ultrasonography. 2019 Jan;38(1):83-91. 
should be aware of common artifacts in order to ensure the accuracy of the diagnosis.

This article describes issues related to the quality of ABUS images and illustrates common artifacts encountered when interpreting ABUS images.

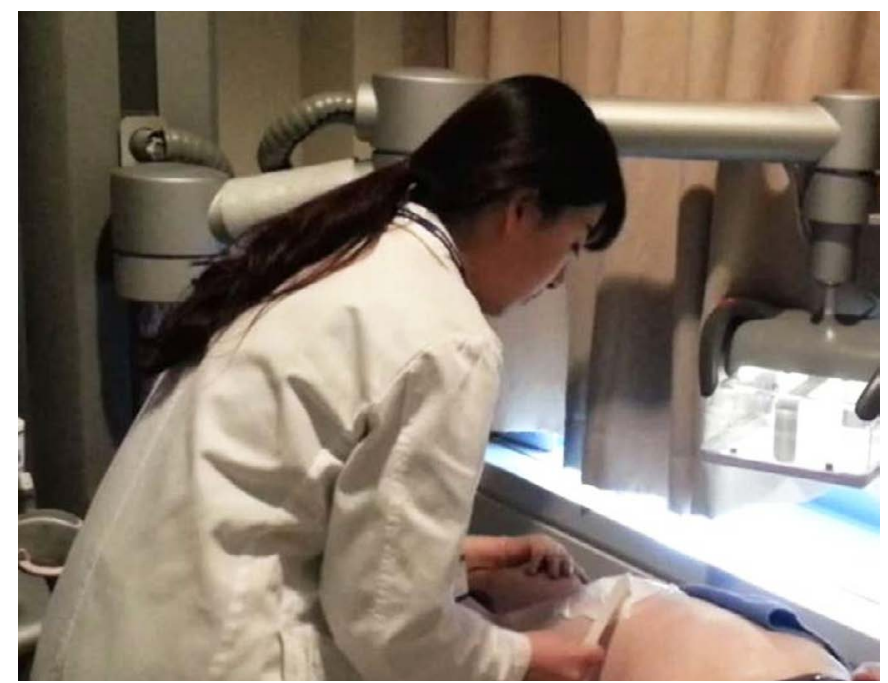

A

Fig. 1. Image acquisition for automated breast ultrasonography.

A. The patient was placed in the supine position. A special lotion was applied to the skin. B. The transducer was positioned on the breast with slight pressure and locked prior to scanning.

\section{Image Acquisition and Image Quality}

According to guidelines on standards of mammography practice, mammography must meet the highest possible quality standards. However, no comparable guidelines and regulations exist for ABUS. The United States Food and Drug Administration recommends 8 hours of training before an operator can use ABUS in clinical settings

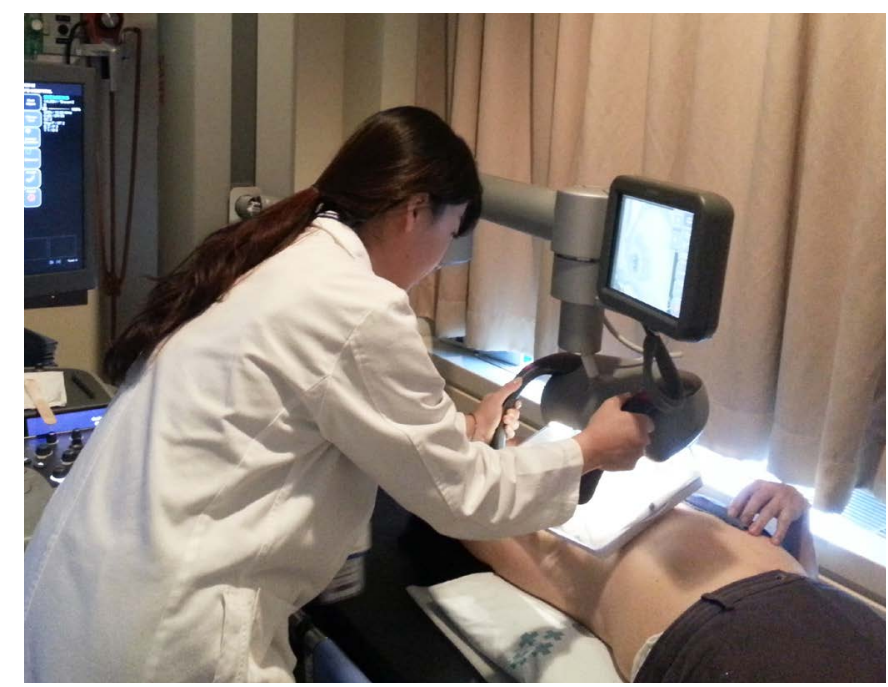

B

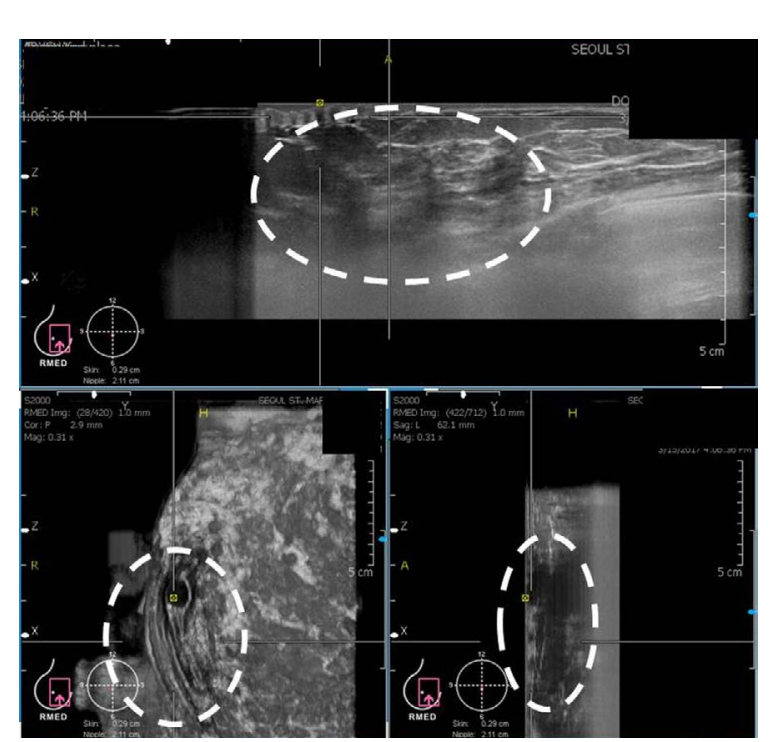

A

Fig. 2. Representative cases showing image quality affected by the acquisition process.

A. A poor-quality image is shown. Compared with the upper breast region, the lower breast is deeply wrinkled, resulting in dirty shadowing in the echo texture (white circles). B. An optimal image is shown. In the anteroposterior view (AP), the nipple is positioned in the center, and in the medial and lateral views (Med, Lat), the nipple is located in the periphery of the image. 
[11].

In ABUS, the patient assumes the same position as in HHUS. An ABUS-exclusive lotion that serves as a coupling agent is applied to the skin instead of the usual gel (Fig. 1A). The transducer is positioned on the breast with slight pressure and locked prior to scanning (Fig. 1B).

The breast tissues should be fully covered. Poor contact between the transducer and skin could prevent the complete coverage of the breast and adversely affect image acquisition (Fig. 2A). Therefore, gentle compression and skillful manipulation of the probe to cover the whole breast is important.

The depth of the scan is specified from $A$ to $D$ (depending on the breast size, as denoted by bra cup size) or from 3.5 to $5 \mathrm{~cm}$ (small, medium, and large), depending on the vendor, and then the scanning area is selected. The probe is placed on the selected area. The breast is scanned and the volume information is acquired.

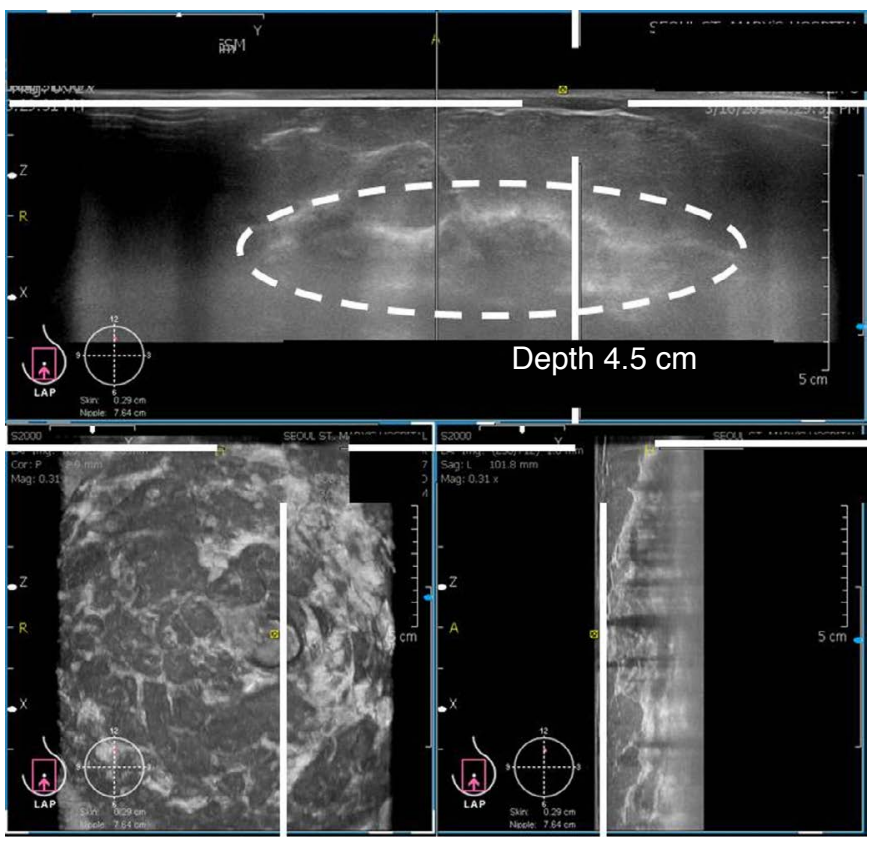

A

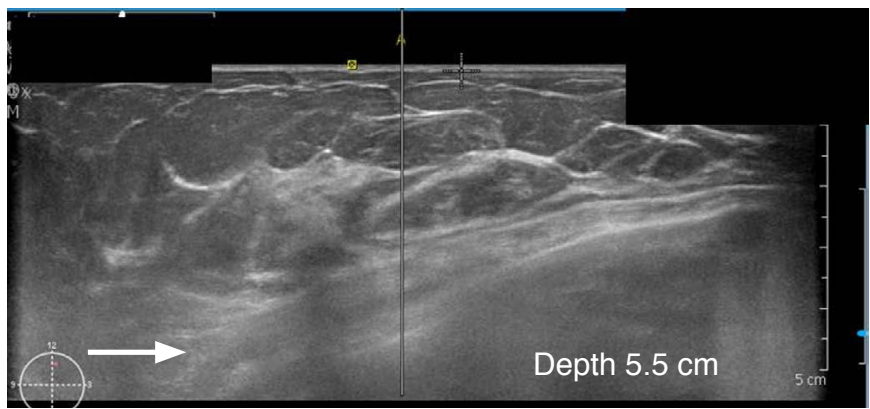

C
Anteroposterior, medial, and lateral views are routinely acquired. If there are additional indications, such as for large breasts, superior and inferior views are also acquired.

In the anteroposterior view, the nipple should be positioned at the center of the image. In the medial and lateral views, the nipple should be positioned at the periphery of the image (Fig. 2B). It is easier to acquire images in women with large breasts than in those with small breasts. The depth of the scan should be evaluated to ensure that the deep and peripheral breast tissues are included in the image fields. In women with large breasts (a bra cup size larger than an E), the upper and deep central breast tissues are frequently not covered. Therefore, additional views should be acquired after correcting for the error in depth (Fig. 3A-C).

When a scan is completed, the coronal plane of the acquired volume is displayed at the skin level. At this level, the technologist can assess the adequacy of the image quality in terms of position,

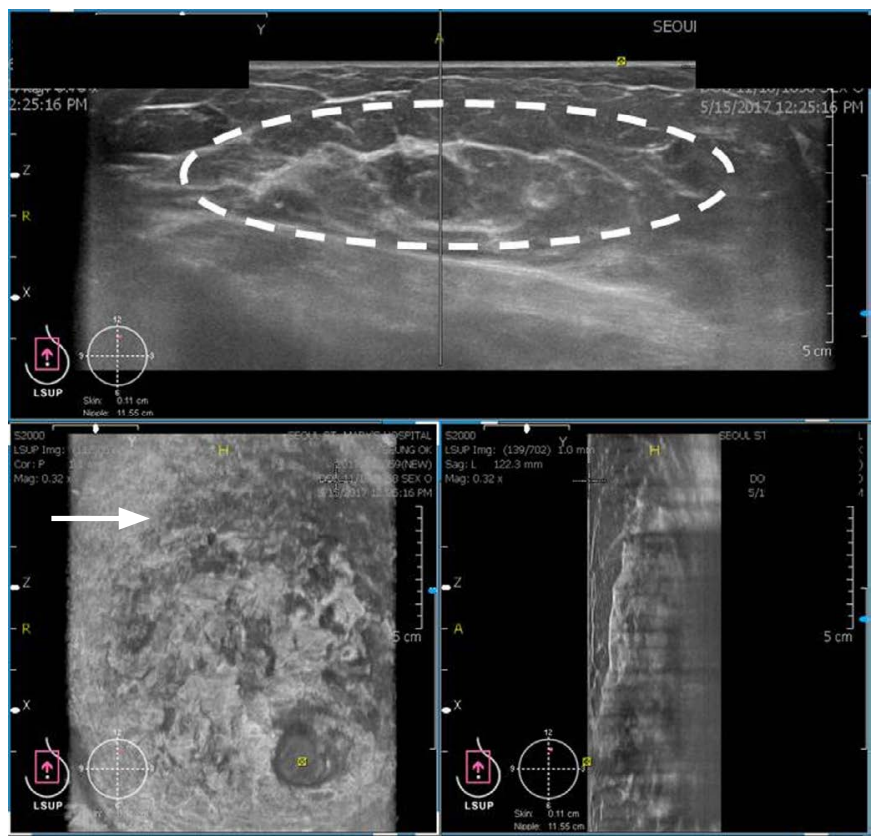

B

Fig. 3. Image depicting depth and coverage in a 44-year-old woman with a bra cup size of $E$.

A. The coronal view shows proper nipple position and coverage, and the axial view still reveals breast tissue (white circle) at the end of the upper breast region. The retromammary fat layer and chest wall are not shown. B. A superior view was additionally obtained. The fat layer without parenchyma (white circle) is shown in the upper breast (arrow). C. Rescanned image with a widened depth of $5.5 \mathrm{~cm}$ includes the chest wall boundary (arrow). 
coverage, and gross contact artifacts (Fig. 4A, B).

\section{Artifacts of ABUS}

Because artifacts are not part of breast tissue anatomy or pathology, and generally interfere with the ability to make an accurate diagnosis, it is important that technologists and radiologists learn to identity and characterize artifacts on ABUS images. Common artifacts encountered in ABUS are described below.

\section{Nipple Shadow and Reverberation Artifacts}

Nipple shadow and reverberation artifacts are problematic in ABUS (Fig. 5).

A reverberation artifact has the typical feature of multiple parallel lines that decrease in intensity and are equidistant from each other [12]. In addition, in the area around the nipple, the contact between the transducer and the skin is often imperfect, resulting in an acoustic shadow behind the nipple.

\section{Skip Artifacts}

A skip artifact is seen as a transverse anechoic line (Fig. 6A). It is common where there is a change in tissue stiffness, either due to a mass or simply due to moving from fat to dense tissue (Fig. 6B).
Although artifacts generally interfere with image quality, this type of artifact can, however, help to characterize a lesion or other findings [13]. When a transverse anechoic line is shown, the radiologist

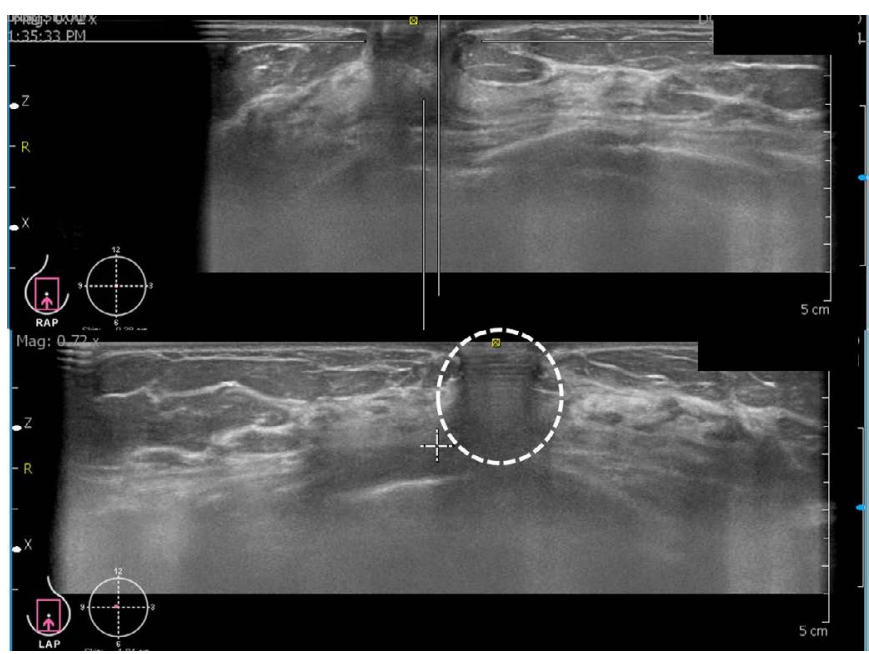

Fig. 5. Nipple shadow and reverberation artifact in a 41-yearold woman. A hypoechoic lesion is shown at the left subareolar region (white circle), in comparison with the right breast (upper image). The patient was recalled for hand-held ultrasonography and no abnormality was seen (not shown). The hypoechoic area was a reverberation artifact showing multiple parallel lines.

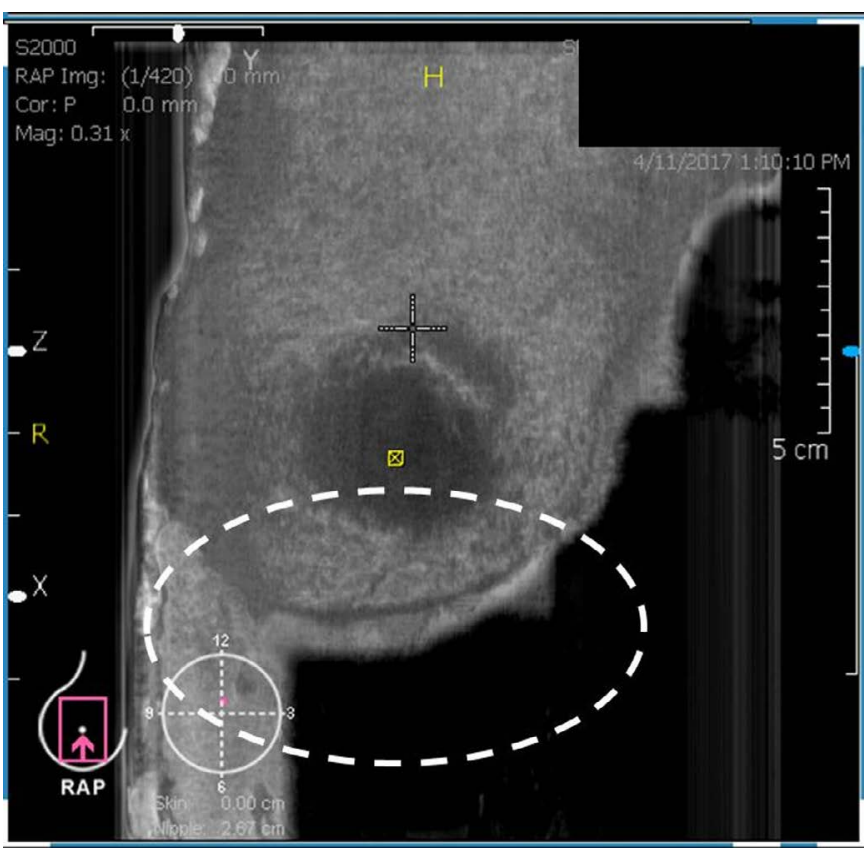

A

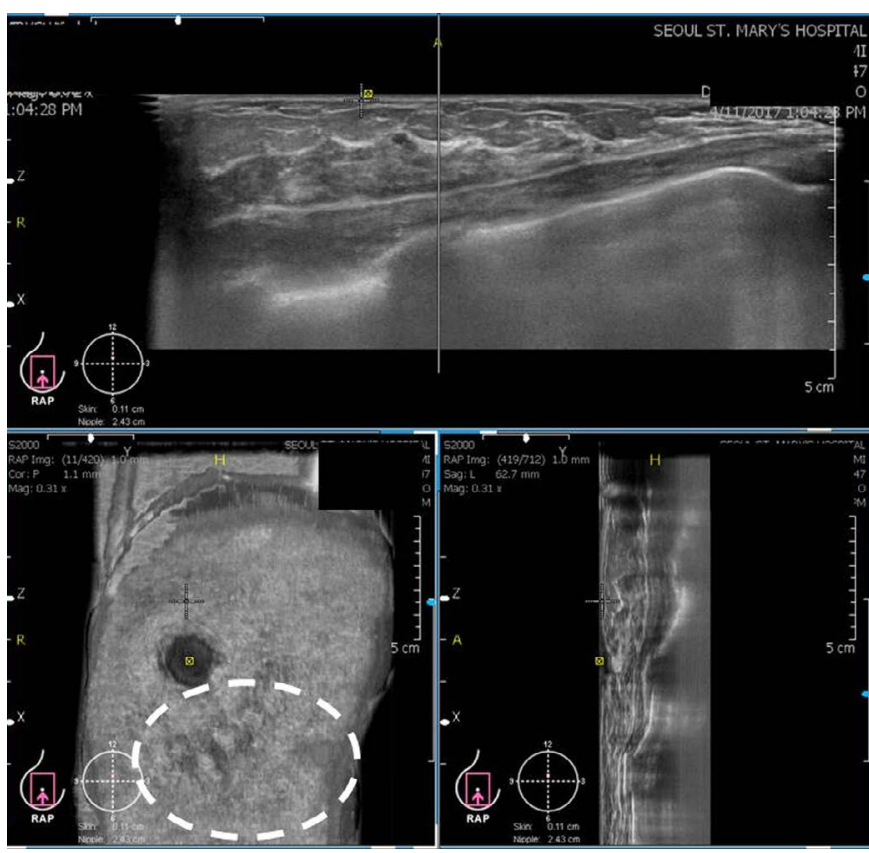

B

Fig. 4. Technologist's assessment of image quality in a 47-year-old woman.

A. After scanning the patient, the coronal plane of the acquired volume was displayed at the skin level. The technologist detected incomplete coverage of the lower and medial breast regions (white circle). B. The probe was repositioned and rescanned. The lower and medial breast was covered adequately (white circle). 


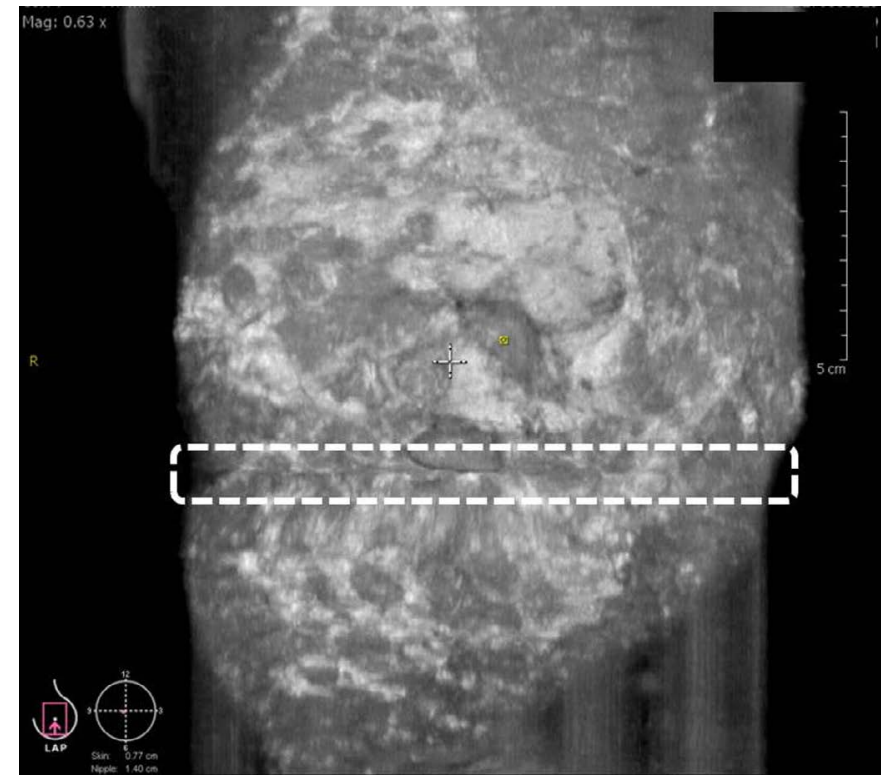

A

Fig. 6. Skip artifact in a 42-year-old woman.

A. Coronal image shows the transverse line (white box). B. On the 3-display view, a true mass is present above this line.

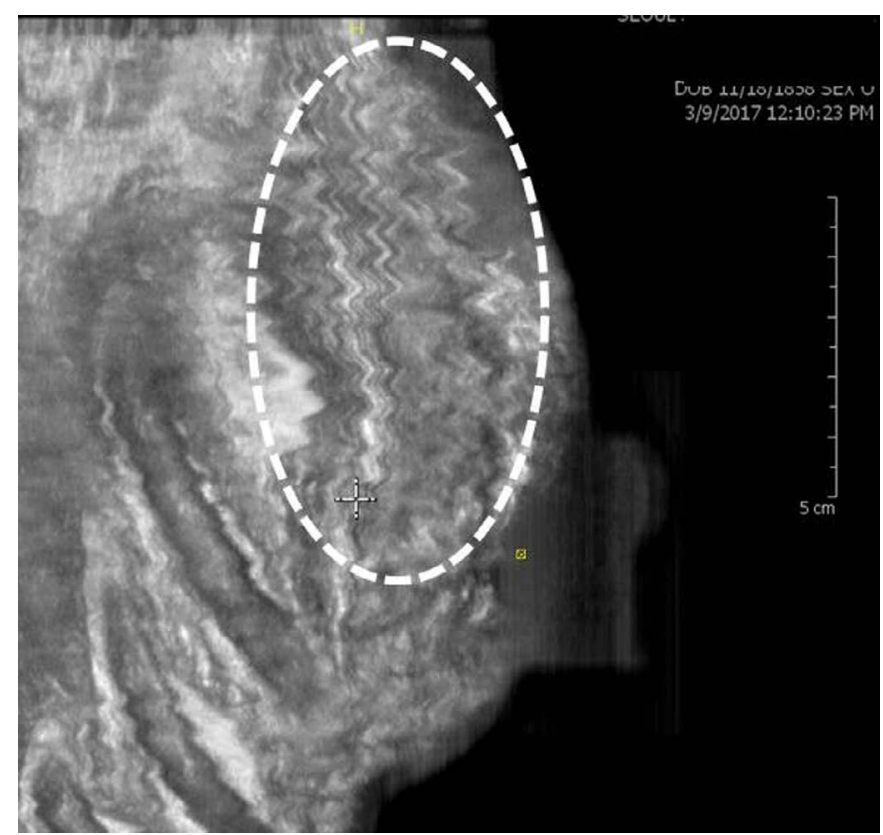

Fig. 7. Motion artifact in a 45-year-old woman. Coronal image shows multiple zigzag lines (white circle).

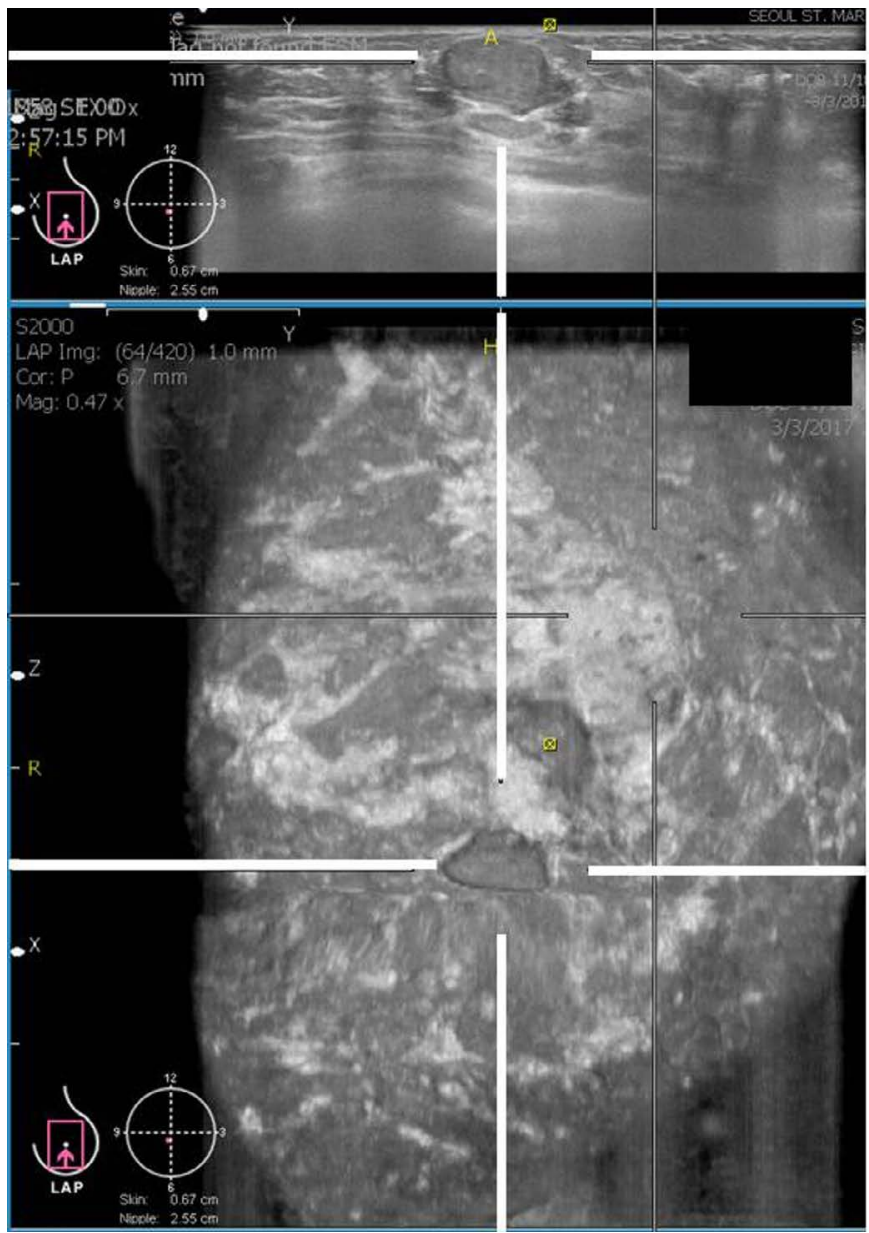

B

must try to identify the cause of the change in tissue stiffness. Skip artifacts can be useful for detecting isoechoic masses, which are difficult to distinguish from the surrounding fat.

\section{Motion Artifacts}

Motion artifacts are seen as multiple zigzag lines. The probe is designed to move smoothly over the area of interest. However, when the probe is placed just above the rib cage and vigorous compression or patient motion occurs, the probe can move discontinuously and create these curly lines (Fig. 7) $[13,14]$.

\section{Contact Artifacts}

A contact artifact appears as an irregular hypoechoic to anechoic lesion depending on the area of poor contact between the transducer and skin (Fig. 8A). It can mimic a breast mass.

The difference between a true lesion and this artifact is that the hypoechoic area of this artifact changes to an echogenic area at the skin layer (Fig. 8B). Contact artifacts occur in the superficial portion of the breast, from the skin layer to the subcutaneous fat layer (Fig. 
8A). However, true lesions are located at the layer of the breast parenchyma (Fig. 8C).

\section{Section Thickness Artifacts}

A section thickness artifact is associated with echo-signal averaging within the section thickness. It is shown as a false area of sludge or debris with an anechoic cystic structure, and is commonly seen in cysts and the bladder (Fig. 9) [12]. Since a wide fixed focal zone is applied in ABUS, these artifacts occur frequently.

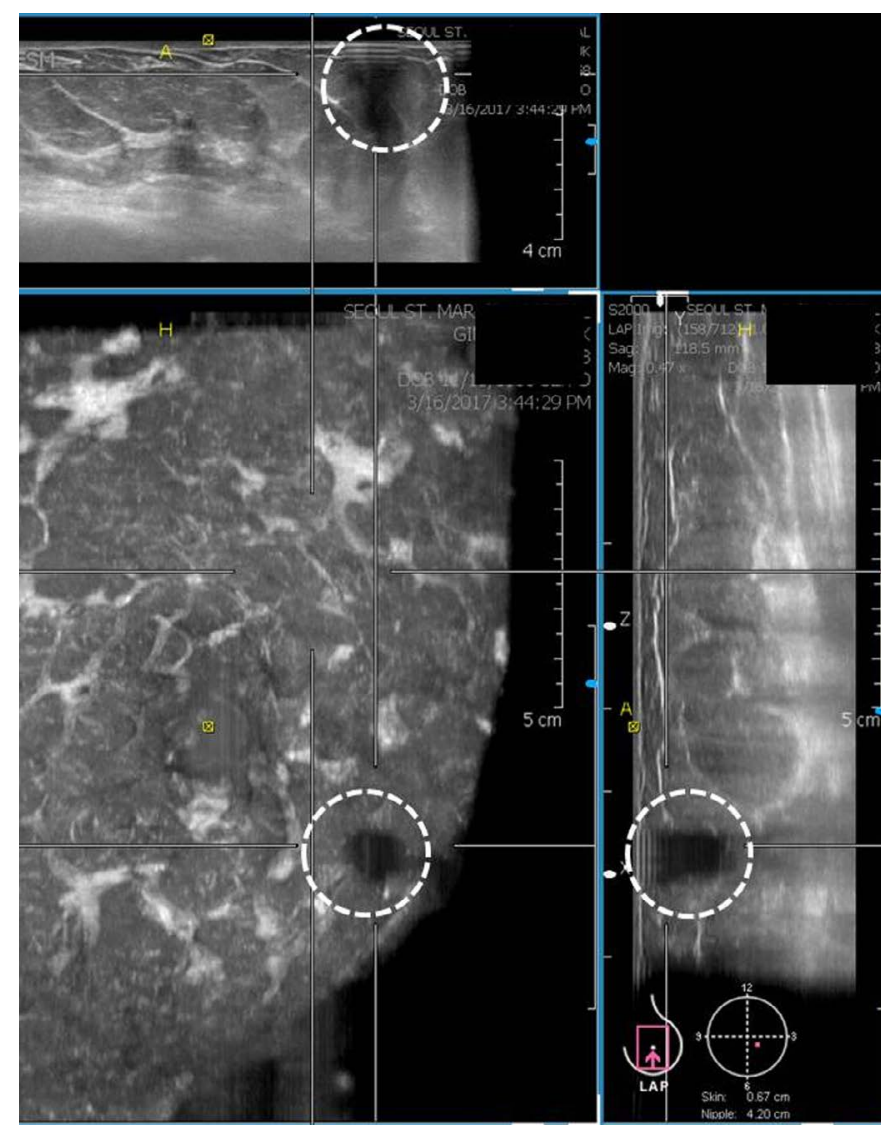

A

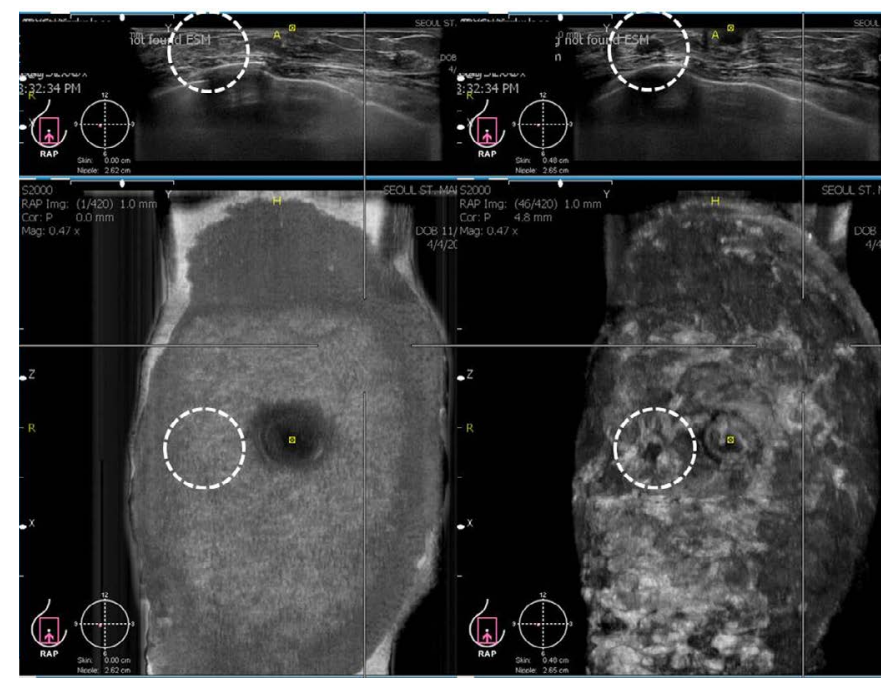

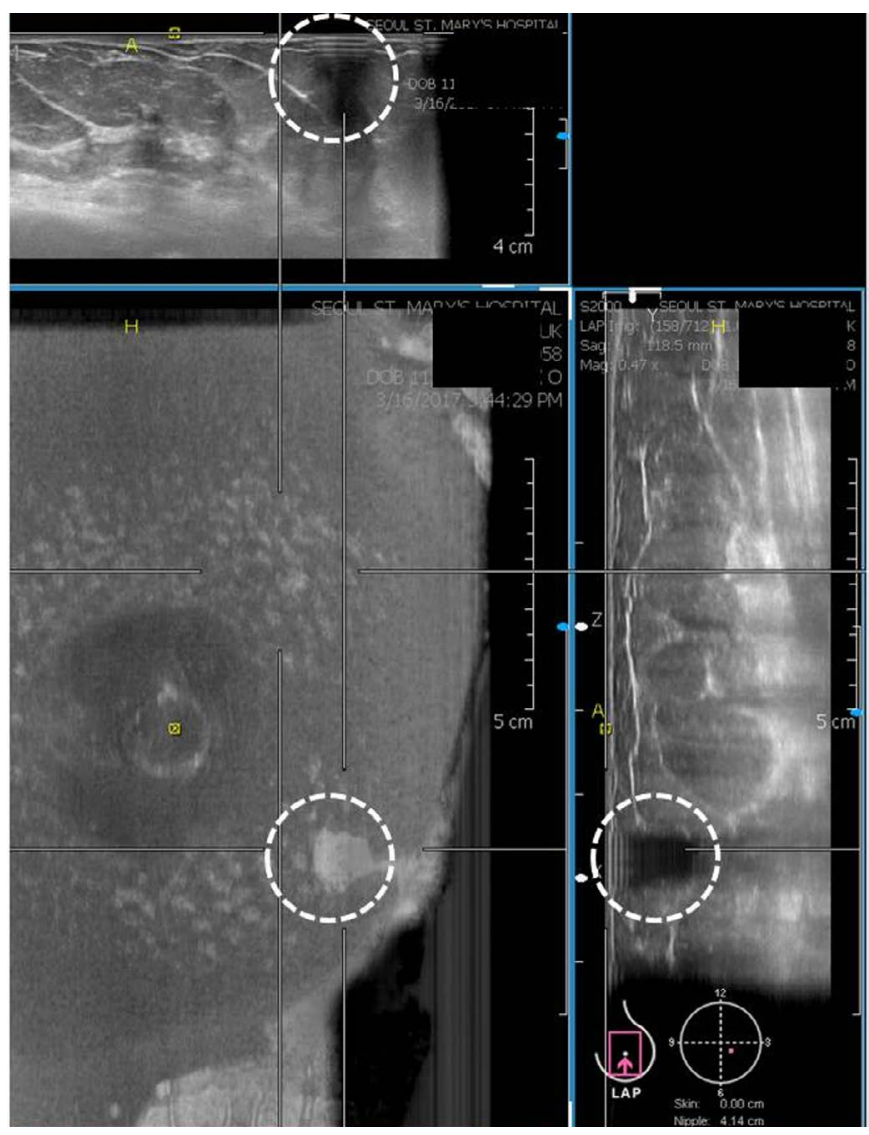

B

Fig. 8. Two representative cases showing a contact artifact and true mass in a 44-year-old woman.

A. An irregular, speculated, hypoechoic lesion (white circles) suspected to be a mass is shown. B. This hypoechoic lesion changed to a white area (white circles) at the skin layer due to poor contact between the transducer and skin. This contact artifact is mainly seen on the superficial breast tissues. C. A true mass (white circles) is located at the layer of the breast parenchyma, at a different point from that of the contact artifact. 


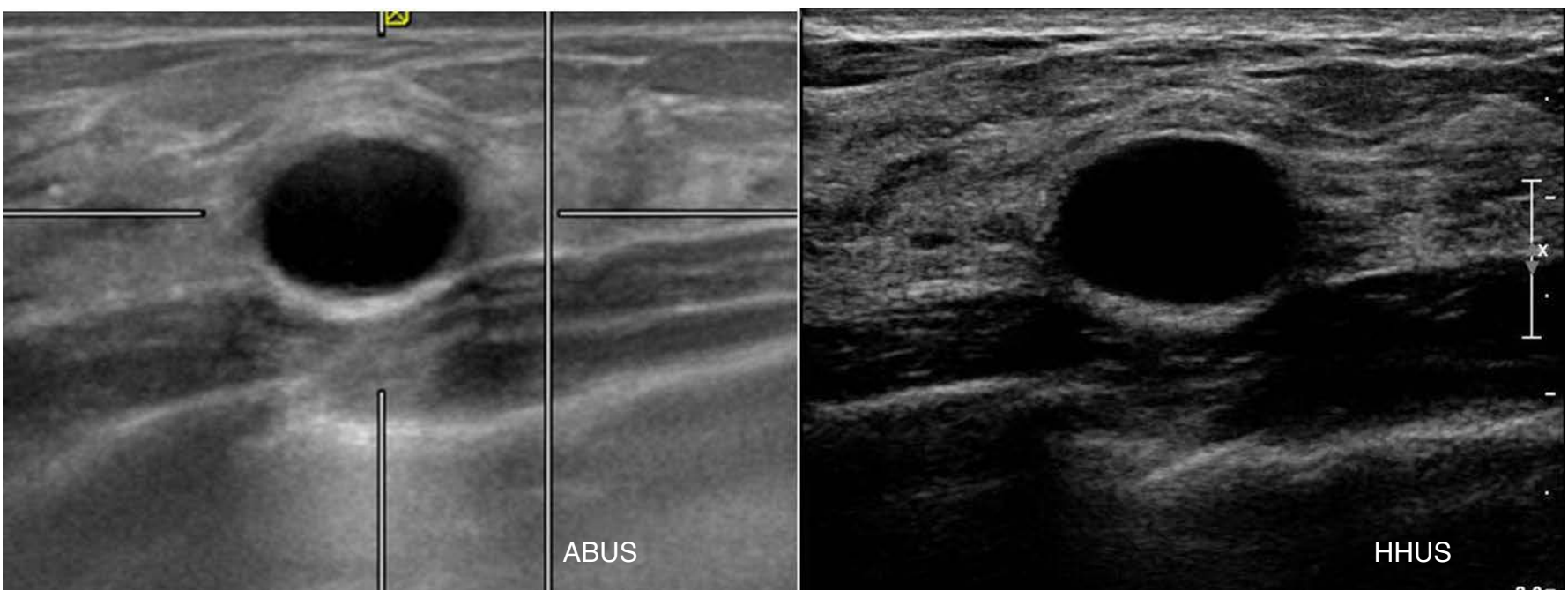

Fig. 9. Section thickness artifact in a 49-year-old woman. Axial image of automated breast ultrasonography (ABUS) demonstrates an anechoic cyst with anterior echogenicity. However, this lesion is shown as a simple cyst on hand-held ultrasonography (HHUS).

\section{How to Improve the Image Quality of ABUS}

Poor image quality in ABUS will ultimately raise questions about its reliability in clinical practice.

In a prospective study [15] comparing the image quality of HHUS and ABUS using identical image pairs of 411 lesions, the image quality of ABUS was identical or superior to HHUS in $97.1 \%$ of cases. However, in $2.9 \%$ of the lesions, ABUS was inferior, and in $0.5 \%$ of cases, the poor quality of ABUS images posed challenges for image interpretation due to incomplete lesion coverage and shadowing by a contact artifact (Fig. 10).

In order to obtain a good image, basic techniques should be followed, including an abundant use of lotion, full coverage and adjustment of depth, gentle compression, and proper transducer placement.

Nipple shadowing and reverberation artifacts are common. To reduce these artifacts, ABUS systems have adopted new processing algorithms [14]. The reverberation removal algorithm determines whether tissue contact is present and removes data corresponding to areas without tissue contact. The adaptive nipple shadow reduction tool analyzes data to enhance the retroareolar structure. Applying abundant lotion often resolves this problem $[9,16]$.

To reduce motion artifacts, the patients should be instructed to breathe superficially and not to talk during the acquisition [16]. Technicians should check for the presence of a contact artifact before the completion of each acquisition and rescan the image if a contact artifact is present [16].

Innovative means of addressing the challenges posed by artifacts should be put forth. A prospective study showed that gel pad application had an effect on scan coverage and image quality

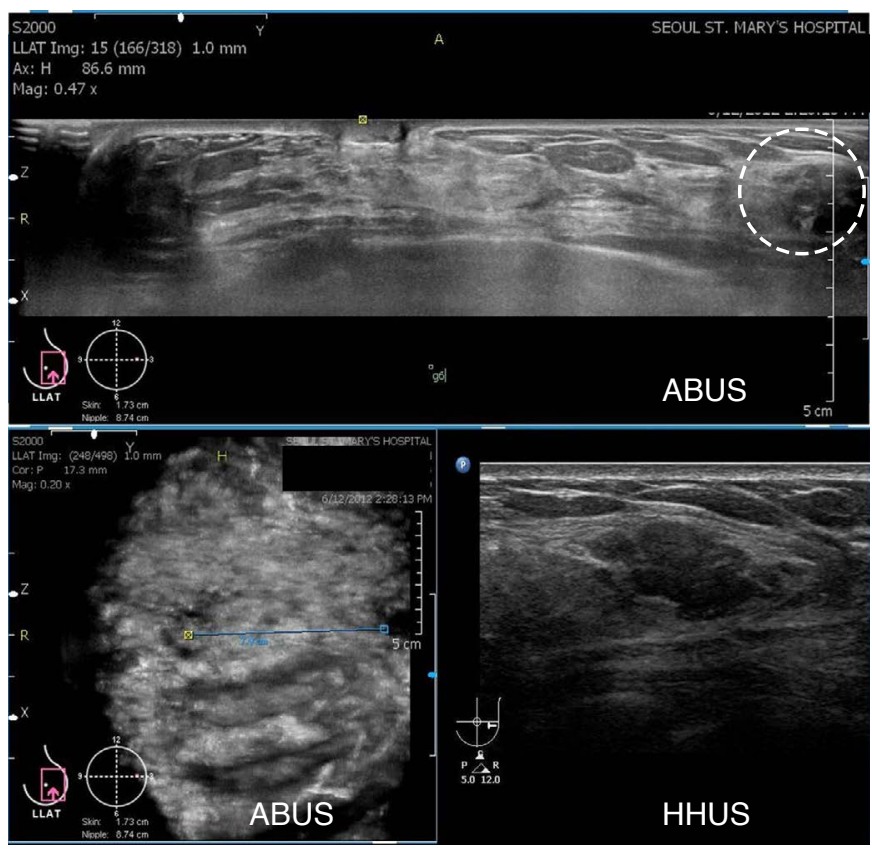

Fig. 10. Incomplete lesion coverage in a 46-year-old woman. A laterally located mass (white circle) was partially included on the lateral view of automated breast ultrasonography (ABUS), hindering the complete characterization of the mass (upper and right lower column). The same mass was fully visualized on hand-held ultrasonography (HHUS, left lower column).

[17]. As the probe moves on the dome-shaped breast horizontally, pressure imbalance and contact defects occur (Fig. 11A). Therefore, image quality can be degraded at the periphery.

A commercial gel pad was used as a specialized coupling agent to improve contact defects (Fig. 11B). As the gel pad was placed on 

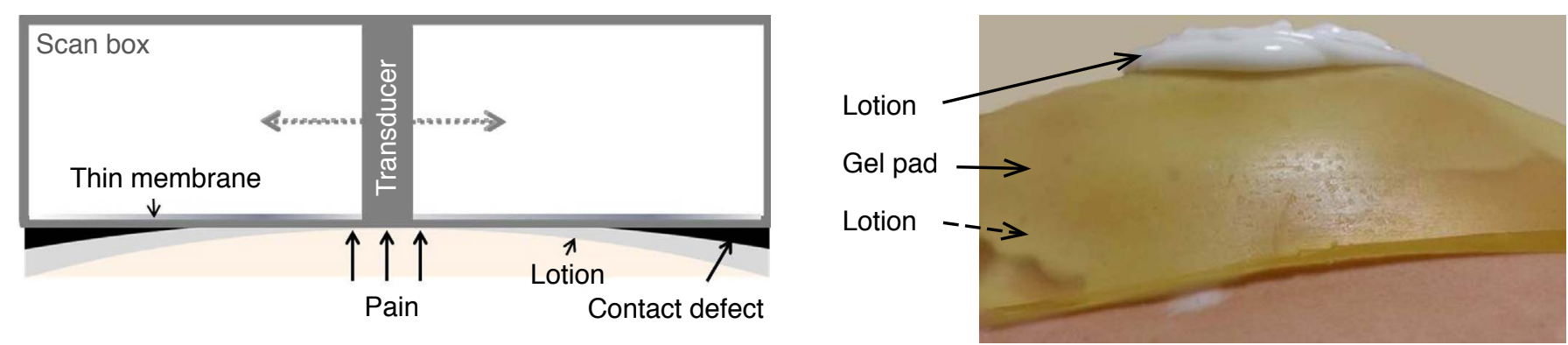

A

B
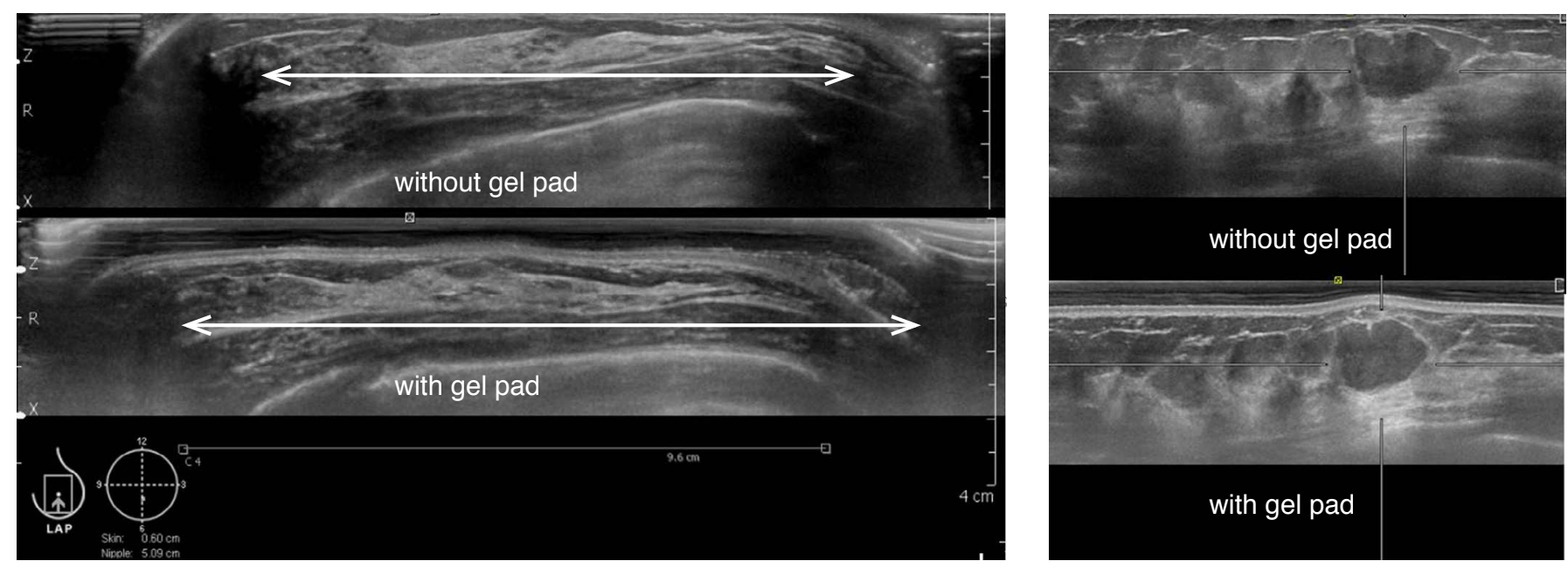

C

D
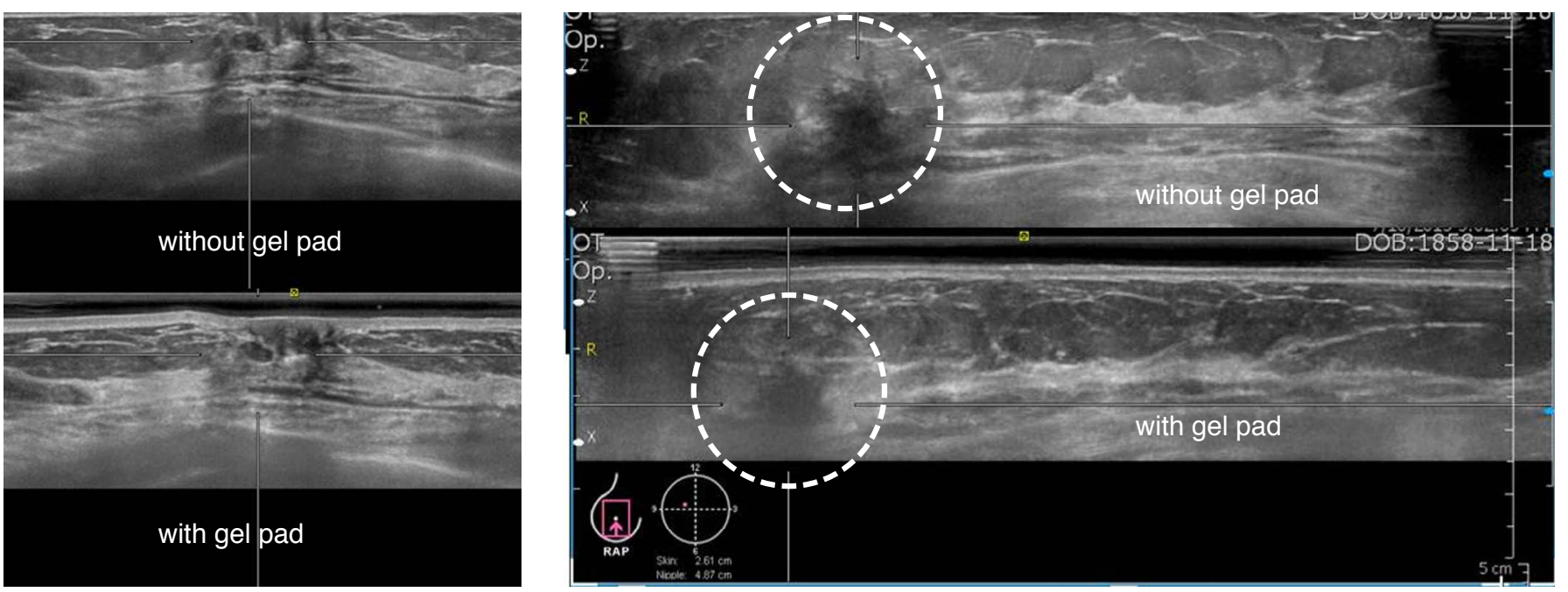

$\mathrm{E}$

Fig. 11. Gel pad application and image quality.

A. Schematic diagram shows a peripheral contact defect. B. A commercial gel pad was applied as a specialized coupling agent. C. The width of the properly scanned area was increased by using a gel pad. D, E. Images with and without a gel pad show no difference in image quality in breast cancer (D) and fibroadenoma (E). F. The deep portion (white circles) was not fully included in the scan field due to the thickness of the gel pad. 
the breast, the breast became more flattened, with fewer contact defects at the periphery. With regard to the coverage of the breast, the area of the breast properly scanned was increased by using a gel pad (Fig. 11C).

In terms of image quality, no significant differences were found between images acquired with or without gel pad application in terms of conspicuity, contrast, degree of retroareolar shadowing, and retroareolar lesion visibility (Fig. 11D, E). When a gel pad is placed on the breast, the deep portion may not be included in the scanning field because of the thickness of the gel pad. Therefore, it is necessary to consider the gel pad thickness when making tissue depth adjustments (Fig. 11F).

In another study, the authors proposed an automated image quality assessment system [9] that evaluated the three aspects of nipple position, nipple shadowing, and breast shape at the point of image acquisition. They applied image processing and machine learning algorithms on ABUS images. The algorithms detected approximately $55 \%$ of images that were rated as low-quality. They concluded that the algorithm was fast and reliable.

\section{Conclusion}

The image quality and diagnostic performance of ABUS and HHUS are comparable. However, in some cases, image artifacts such as nipple shadowing, reverberation artifacts, and improper acquisition might be obstacles to the optimization of image quality. Hence, radiologists and technologists need to be familiar with these image defects and how to reduce and utilize them, where possible, for proper diagnoses to be made in clinical settings.

ORCID: Sung Hun Kim: https://orcid.org/0000-0003-4478-9720

\section{Conflict of Interest}

No potential conflict of interest relevant to this article was reported.

\section{References}

1. Shapiro S. Screening: assessment of current studies. Cancer 1994;74(1 Suppl):231-238.

2. Kerlikowske K, Hubbard RA, Miglioretti DL, Geller BM, Yankaskas $B C$, Lehman $C D$, et al. Comparative effectiveness of digital versus film-screen mammography in community practice in the United
States: a cohort study. Ann Intern Med 2011;155:493-502.

3. Freer PE. Mammographic breast density: impact on breast cancer risk and implications for screening. Radiographics 2015;35:302315.

4. Kaplan SS. Automated whole breast ultrasound. Radiol Clin North Am 2014;52:539-546.

5. Kotsianos-Hermle D, Wirth S, Fischer T, Hiltawsky KM, Reiser M. First clinical use of a standardized three-dimensional ultrasound for breast imaging. Eur J Radiol 2009;71:102-108.

6. Kelly KM, Dean J, Comulada WS, Lee SJ. Breast cancer detection using automated whole breast ultrasound and mammography in radiographically dense breasts. Eur Radiol 2010;20:734-742.

7. Kim SH, Kang BJ, Choi BG, Choi JJ, Lee JH, Song BJ, et al. Radiologists' performance for detecting lesions and the interobserver variability of automated whole breast ultrasound. Korean J Radiol 2013;14:154-163.

8. Shin HJ, Kim HH, Cha JH. Current status of automated breast ultrasonography. Ultrasonography 2015;34:165-172.

9. Schwaab J, Diez Y, Oliver A, Marti R, van Zelst J, Gubern-Merida A, et al. Automated quality assessment in three-dimensional breast ultrasound images. J Med Imaging (Bellingham) 2016;3:027002.

10. Odle TG. Breast imaging artifacts. Radiol Technol 2015;87: $65 \mathrm{M}-87 \mathrm{M}$.

11. Mendelson EB, Berg WA. Training and standards for performance, interpretation, and structured reporting for supplemental breast cancer screening. AJR Am J Roentgenol 2015;204:265-268.

12. Baad M, Lu ZF, Reiser I, Paushter D. Clinical significance of US artifacts. Radiographics 2017;37:1408-1423.

13. Dubelaar I, Fassaert T, de Jong MD, Ruttern M. Automated breast volume scanning: technique and artefacts. In: European Congress of Radiology 2011; 2011 Mar 3-7; Vienna, Austria; poster No. C-1539.

14. Gazhonova V. 3D automated breast volume sonography. Cham: Springer, 2017.

15. An YY, Kim SH, Kang BJ. The image quality and lesion characterization of breast using automated whole-breast ultrasound: a comparison with handheld ultrasound. Eur J Radiol 2015;84:1232-1235.

16. van Zelst JCM, Mann RM. Automated three-dimensional breast US for screening: technique, artifacts, and lesion characterization. Radiographics 2018;38:663-683.

17. Kim YJ, Kim SH, Jeh SK, Choi JJ, Kang BJ, Song BJ. Gel pad application for automated breast sonography. J Ultrasound Med 2015;34:713-719. 